\author{
Cadernos de \\ ESTUDOS LINGḯĺSTICOS - (55.1), Campinas, Jan./Jun. 2013
}

\title{
A SEMÂNTICA DE FRAMES COMO PARADIGMA PARA OS ESTUDOS MULTILÍNGUES: O CASO DOS FRAMES SELF MOTION E CRIMINAL_PROCESS EM INGLÊS E PORTUGUÊS $\bar{S}^{1}$
}

\author{
ROVE CHISHMAN ${ }^{2}$ \\ ANDERSON BERTOLDI ${ }^{3}$
}

\begin{abstract}
RESUMO
Este artigo tem por finalidade discutir a forma como a descrição do significado lexical proposto pela Semântica de Frames pode beneficiar os estudos multilíngues, principalmente no que se refere ao estabelecimento de equivalentes em diferentes línguas. Para tal, apresentamos duas análises contrastivas: uma explorando as unidades lexicais do frame Self motion e outra destacando oframe Criminal_process. No que se refere aos verbos de movimento, constatamos que a polissemia do verbo to walk na língua-fonte pode ser constatada por meio da diversidade de equivalentes na língua portuguesa: caminhar, andar e passear. Pudemos também fazer uma aproximação entre a proposta de Talmy (1985) e o arcabouço da FrameNet, ao constatar que, no Português, os verbos que incorporam maneira de movimento na raiz do verbo são em número bem inferior ao que se constata no Inglês. No caso das unidades lexicais relacionadas ao domínio jurídico, as diferenças entre as realidades culturais geram diferentes graus de equivalência. As unidades lexicais to charge, em Inglês, e acusar, em Português, podem ser considerados equivalentes. No entanto, o conhecimento jurídico expresso por ambas as unidades lexicais é diferente. O evento jurídico representado pela unidade to charge no sistema jurídico americano não corresponde plenamente ao evento jurídico representado pela unidade acusar. Já no caso da unidade lexical arraignment, o evento jurídico expresso pela unidade lexical é tão típico do sistema jurídico norte-americano que não possui equivalente em Português. A Semântica de Frames ajuda a contrastar as diferentes realidades culturais que são evocadas pelas unidades lexicais em diferentes línguas.
\end{abstract}

Palavras-chaves: Semântica de Frames; estudos multilíngues; análise contrastiva.

\section{ABSTRACT}

This paper discusses the use of Frame Semantics for lexical meaning description in multilingual studies, especially to identify equivalents in different languages. The frames Self motion and Criminal_process exemplify the use of Frame Semantics as a paradigm for multilingual studies. Concerning motion verbs, the polysemy of the verb to walk is expressed in Portuguese by a great variety of equivalents, such as: caminhar, andar and passear. This paper allies the lexicalization patterns study of Talmy (1985) to the FrameNet paradigm. In Portuguese, the number of verbs that conflate motion and manner in verb roots are lesser than in English, comparing those verbs that evoke Self_motion frame in FrameNet to their equivalents in Portuguese. In the case of lexical units of the legal domain, differences in cultural realities cause different levels of cross-lingual equivalence. Lexical units to charge in English and acusar in Portuguese are considered as equivalents. However,

\footnotetext{
1. Este estudo contou com apoio do CNPq, CAPES e FAPERGS.

2. UNISINOS (RS)/CNPq/Pq, Brasil. rove.chishman@gmail.com

3. UNISINOS (RS), Brasil. andersonbertoldi@yahoo.com
} 
CHISHMAN e BERTOLDI - A semântica de frames como paradigma...

the legal knowledge evoked for both lexical units are not equivalent. The legal event represented by the lexical unit to charge in the American legal system does not fully correspond to the legal event represented by the lexical unit acusar. In the case of the lexical unit arraignment, the legal event represented by the lexical unit is so peculiar to the American legal system that it does not present an equivalent in Portuguese. Frame Semantics may be used as a paradigm to contrast the differences in the cultural realities that are evoked by lexical units in different languages.

Key-words: Frame Semantics; multilingual studies; contrastive analysis.

\section{INTRODUÇÃO}

O interesse no uso da Semântica de Frames (FILLMORE, 1982; 1985) como paradigma para estudos multilíngues tem aumentado na última década. Esse crescente interesse se deve, em grande parte, ao sucesso alcançado pela base de dados online FrameNet (FILLMORE; JOHNSON; PETRUCK, 2003), que atualmente conta com mais de 900 frames semânticos descritos para a representação do significado dos itens lexicais da língua inglesa. A FrameNet é uma base de dados lexicais desenvolvida segundo os princípios da Semântica de Frames. Seguindo esses princípios, a FrameNet descreve o significado dos itens lexicais por meio da relação de unidades lexicais a estruturas esquematizadas de conhecimento chamadas frames.

O desenvolvimento de bases de dados similares à FrameNet para línguas como o alemão (BOAS, 2002), o espanhol (SUBIRATS, 2009), o japonês (OHARA, 2009) e o português (SALOMÃO, 2009), entre outras, tem estimulado a discussão sobre o uso da Semântica de Frames e da FrameNet como paradigma para a análise linguística multilíngue 4 . A polissemia das línguas, os diferentes padrões de valência e de lexicalização das línguas, bem como as relações de paráfrase e de equivalência de tradução são os grandes desafios a serem enfrentados pelos estudos multilíngues, especialmente tratando-se de criação de recursos lexicais multilíngues (BOAS, 2005). Assim, qualquer estudo multilíngue visando à criação de recursos lexicais, seja uma base de dados lexicais seguindo o paradigma da FrameNet, seja um corpus com anotação semântica de frames, enfrentará o desafio da equivalência entre itens lexicais de línguas diferentes.

A associação da Semântica de Frames aos estudos multilíngues não é recente. Os conceitos de cenas e frames (FILLMORE, 1977) têm sido utilizados nos estudos de tradução desde a década de 80 (VANNEREM; SNELL-HORNBY, 1986; SNELL-HORNBY, 1988; 2005; VERMEER; WITTE, 1990). Mais recentemente, a análise contrastiva, que inicialmente era voltada ao ensino de línguas, tem utilizado a Semântica de Frames como paradigma para comparação linguística, com vistas à criação de recursos léxico-computacionais baseados em frames, como mostra a coletânea de trabalhos editados por Boas (2009).

4. O termo multilíngue, tal como está sendo utilizado neste artigo, diz respeito ao estudo de mais de uma língua. Não se está fazendo diferenças entre estudos bilíngues e multilíngues. Pelo contrário, utiliza-se, aqui, a expressão multilíngue englobando a ideia de aplicação bilíngue e multilíngue sob o mesmo rótulo. 
Este artigo tem por finalidade discutir a forma como a descrição do significado lexical proposto pela Semântica de Frames pode beneficiar os estudos multilíngues, principalmente no que toca o estabelecimento de equivalentes em diferentes línguas. Para tratar desse tema, este trabalho está organizado como segue. Na seção 2 será discutida a problemática da equivalência nos estudos multilíngues. Na seção 3 será abordada a teoria dos frames semânticos de Charles J. Fillmore e sua aplicação aos estudos multilíngues. Na seção 4 será apresentado um estudo contrastivo do frame Self motion. Na seção 5 será apresentado um estudo contrastivo do frame Criminal_process. Na seção 6, discute-se como a Semântica de Frames, através da sua abordagem cognitiva do significado, pode auxiliar nos estudos contrastivos. Por fim, na seção 7, serão apresentadas as considerações finais deste trabalho, especialmente no que tange ao uso da Semântica de Frames como perpectiva teórica para análise contrastiva do léxico.

\section{A PROBLEMÁticA DA EQUIVALÊNCIA NOS ESTUDOS MULTILÍNGUES}

A equivalência é um desafio para os estudos multilíngues. Tanto a tradução quanto a lexicografia ou a análise contrastiva necessitam estabelecer equivalentes na língua-alvo para os itens lexicais da língua-fonte. Encontrar um equivalente na língua-alvo para um item lexical da língua-fonte não é uma prática trivial. Nem sempre um item lexical em uma língua possui um equivalente em outra língua. Outras vezes, os padrões de polissemia das línguas são tão variáveis que um item lexical em uma língua pode possuir diversos equivalentes em outra língua, ou diferentes itens lexicais em uma língua apresentarem somente um equivalente em outra língua. Essa dificuldade de estabelecer equivalentes em diferentes línguas pode representar um obstáculo para a tradução, por exemplo.

Pode-se ilustrar essa dificuldade em se estabelecer equivalentes em diferentes línguas por meio do verbo inglês to walk (caminhar, andar, passear). O Longman Dictionary of Contemporary English (LDCE) (1995, p. 1605) traz a seguinte definição para o verbo to walk: "1 (...) to move along putting one foot in front of the other (...). 2 (...) to walk in order to get somewhere, across a particular area or distance (...)." Além da definição para o verbo to walk, o LDCE traz diferentes expressões idiomáticas: walk the dog (levar o cachorro para caminhar), walk somebody home/walk somebody to school (levar alguém para casallevar alguém para a escola), go walking (fazer caminhada).

Essa diversidade de sentidos é expressa pelas equivalências apresentadas pelos dicionários bilíngues para o verbo to walk. O Dicionário Inglês-Português (HOUAISS; CARDIM, 2000, p. 864) apresenta os seguintes equivalentes para o verbo to walk: "andar por, percorrer, atravessar (a pé); andar sobre; caminhar; levar a passeio (cavalo, cachorro); fazer andar; fazer andar a passo (cavalo);

5. “1 (..) mover-se pondo um pé na frente do outro (...). 2 (...) andar a fim de chegar a algum lugar, percorrendo uma distância ou área particular (...).” 
CHISHMAN e BERTOLDI - A semântica de frames como paradigma...

levar, conduzir (fazendo caminhar) (...)." Um dos desafios, portanto, consiste em explicar a diversidade de equivalentes apresentada pelos dicionários bilíngues e as dificuldades em se estabelecer a equivalências entre línguas Para responder a essa questão, apresenta-se a abordagem de três diferentes autores para a equivalência.

Altenberg e Granger (2002) seguem uma abordagem fundamentada na linguística contrastiva. A proposta desses autores é baseada nas relações que os itens lexicais estabelecem entre as diferentes línguas, considerando o nível de sobreposição de significados de um item lexical entre a língua-fonte e a línguaalvo. Para Altenberg e Granger (2002), as diferentes extensões de significados nas línguas dão origem a diferenças nos processos de lexicalização, de gramaticalização e de extensão metafórica, o que resulta em padrões complexos de polissemia nas línguas. Assim, Altenberg e Granger (2002, p. 22) distinguem entre três tipos de relações entre línguas, baseados na correspondência dos padrões de polissemia entre as línguas:

a) a polissemia sobreposta ocorre quando os itens lexicais em duas línguas possuem basicamente as mesmas extensões de significado. No entanto, essa completude de correspondência é muito difícil de existir. Ex.: computador/ computer, oxigênio/oxygen;

b) a polissemia divergente ocorre quando os itens lexicais em duas línguas têm diferentes extensões de significado. Segundo Altenberg e Granger (2002), os casos em que uma única palavra em uma língua corresponde a diversas palavras em outra língua é uma variante da polissemia divergente. Ex: walk/caminhar, andar, passear;

c) a correspondência inexistente é a falta de um correspondente na línguaalvo para um item lexical da língua-fonte. Ex.: arraignment, hike, wade.

O trabalho de Snell-Hornby tem como base os estudos de tradução. As relações de equivalência propostas Snell-Hornby (1983) têm como base o seu estudo sobre a correspondência dos verbos em inglês e alemão. Snell-Hornby (1983) propõe quatro níveis de equivalência, baseadas, não nas relações que os itens lexicais estabelecem entre as línguas, mas no núcleo semântico de cada item lexical:

a) a equivalência total implica uma correspondência intensional e extensional de dois itens lexicais em línguas diferentes. Esse é o caso de termos técnicos, como oxygen/oxigênio, e termos de difusão internacional, como computador/computer ou typewriter/máquina de escrever;

b) a equivalência de trabalho envolve casos em que um item lexical em uma língua possui diversos correspondentes em outra língua, no entanto, o equivalente necessitará ser encontrado com base no contexto. Esse é o caso do verbo to know em inglês, que em português possui dois correspondentes, saber e conhecer;

c) a cobertura parcial envolve casos em que um item lexical da línguafonte possui correspondência parcial na língua-alvo. Assim, parte do núcleo do significado do verbo na língua-fonte apresenta um correspondente na língua-alvo 
e o restante do significado necessita ser expresso por paráfrase. Esse é o caso do verbo inglês tiptoe; cujo significado de movimento encontra correspondente em um verbo e a maneira como o movimento é realizado necessita ser parafraseada: caminhar na ponta dos pés ou andar na ponta dos pés;

d) a cobertura-zero ocorre quando um determinado item lexical expressa um conhecimento cultural muito especifico. Esse item tende a não encontrar correspondência. Esse é o caso de alguns termos jurídicos em inglês, como arraignment.

A equivalência também pode ser vista sob o viés do conhecimento cultural e da distinção entre valor denotativo e conotativo. Segundo Duval (2008 [1991]), os casos mais simples de equivalência são aqueles em que “(...) o significado aponta para a mesma realidade cultural e quando o significante é um item reconhecido no léxico de ambas as línguas (p. 274, tradução nossa)." ${ }^{\circ}$ Como exemplo, pode-se citar computador/computer, máquina de escrever/typewriter ou oxigênio/oxygen.

Em outros casos, o item lexical está presente no léxico de ambas as línguas. No entanto, a realidade cultural representada pelo item na língua-fonte não faz parte do conhecimento do falante da língua-alvo. Um exemplo dessa realidade cultural expressa por um item da língua-fonte é the 4th of July (4 de julho). Apesar de the 4th of July ter equivalente em português, a expressão 4 de julho em português não expressa a realidade cultural americana. No contexto cultural americano, a expressão the 4th July não é apenas um dia do ano, mas significa o dia da independência americana.

Há ainda casos em que tanto o item lexical quanto a realidade cultural que ele representa só existem na língua-fonte. Esse é o caso do termo jurídico arraignment, que não possui equivalente em português. Nesses casos, o dicionário necessita criar uma paráfrase para explicar o significado do item na língua-fonte.

Duval (2008 [1991]) ainda afirma que "a equivalência perfeita exige níveis iguais de denotação, por exemplo, a referência ao mesmo elemento da realidade, e níveis iguais de conotação, por exemplo, a referência à mesma rede de associações culturais relacionadas às palavras em ambas as línguas (p.275). ${ }^{7}$ " Assim, itens lexicais como cardiology e cardiologia possuem o mesmo valor denotativo e conotativo. Já o item lexical tíbia possui dois possíveis equivalentes em inglês: tibia e shinbone. Embora esses equivalentes possuam o mesmo valor denotativo, eles não compartilham o mesmo valor conotativo. Enquanto tibia é utilizado em contextos mais formais ou científicos, shinbone é utilizado em contextos mais informais ${ }^{8}$.

6. “(...) the signifie points to the same cultural reality and when the significant is a recognized item in the lexicon of both languages."

7. "Perfect equivalence demands equal levels of denotation, i.e. reference to the same element of the real, and equal levels of connotation, i.e. reference to the same network of cultural associations linked to the words in both languages."

8. O exemplo de tibia e shinbone foi extraído de Duval (2008 [1991]), p. 275. 
CHISHMAN e BERTOLDI - A semântica de frames como paradigma...

Nas seções seguintes, será abordada a forma como a equivalência pode ser tratada sob o viés da Semântica de Frames.

\section{A SEMÂNTICA DE FRAMES}

Uma das perspectivas teóricas para definir classes verbais parte do princípio de que os significados das palavras devem ser descritos em relação a frames semânticos, isto é, "representações esquemáticas das estruturas conceituais e padrões de crenças, práticas, instituições, imagens, etc., que fornecem a base para as interações em uma determinada comunidade" (FILLMORE; JOHNSON; PETRUCK, 2003, p. 235). ${ }^{9}$ De acordo com a Semântica de Frames (FILLMORE, 1982; 1985), a unidade primária de análise, no nível da palavra, é a unidade lexical (cf. CRUSE, 1986), que consiste no pareamento de uma palavra com um sentido. Cada sentido é descrito em relação ao frame semântico que evoca. Por exemplo, o verbo falar, segundo esta ótica, pode ser associado a, pelo menos, duas unidades lexicais distintas, cada uma evocando um frame distinto. Um dos frames é o frame Statement e o outro é oframe Spelling_and_pronoucing.

Um exemplo clássico que Fillmore (1985) apresenta para justificar essa forma de organizar os conceitos é o frame transação comercial, incluindo elementos como comprador, vendedor, mercadorias e dinheiro. É mister observar que estes elementos são apontados como papéis situacionais, e não como papéis semânticos, tal como previsto pela gramática de casos (FILLMORE, 1968). Entre os verbos semanticamente associados a este frame temos comprar, vender, pagar, gastar, custar e trocar, cada um evocando diferentes aspectos do frame. O verbo comprar, por exemplo, põe em perspectiva o comprador e a mercadoria, deixando o vendedor e o dinheiro em segundo plano.

Petruck (2004), referindo-se ao exemplo acima, chama a atenção para mais um aspecto a ser contemplado pela Teoria dos Frames Semânticos: a descrição das propriedades gramaticais. Isso significa que uma descrição completa de verbos que compõem um frame deve incluir o conjunto completo de padrões sintáticos em que ocorrem. Os estudos empreendidos no âmbito do Projeto FrameNet contemplam este compromisso, à medida que explicitam não apenas os padrões semânticos, mas os sintáticos.

Contudo, uma das principais diferenças entre a Semântica de Frames e outras abordagens que também se voltam para as propriedades gramaticais dos verbos, especialmente as que tratam de alternâncias verbais (RAPPAPORT; LEVIN, 1988) ou de construções (GOLDBERG, 1995; 2002), diz respeito ao fato de que a Semântica de Frames (como implementada na FrameNet ${ }^{10}$ ) assume que os sentidos são divididos, em vez de considerar que um dos sentidos é mais básico, derivando

9. “(...) schematic representations of the conceptual structures and patterns of beliefs, practices, institutions, images, etc. that provide a foundation for meaningful interaction in a given speech community."

10. Maiores informações em http://framenet.icsi.berkeley.edu) 
os demais. Uma das vantagens em se descreverem várias unidades lexicais de uma palavra e seus respectivos frames, conforme enfatiza Boas (2005), está em se obter um nível mais preciso de descrição. Evita-se também a necessidade de se adotarem distinções sintáticas como a base para o estabelecimento de um inventário de categorias semânticas.

O modelo dos frames semânticos também é apresentado por Fillmore como expressando sua insatisfação em relação às demais abordagens semânticas. Ao descrever seu modelo semântico como um modelo da semântica da compreensão (semantics of understanding) ou U-semantics (FILLMORE, 1985), Fillmore expressa sua crítica a duas perspectivas distintas: a semântica das condições de verdade, identificada por ele como T-semantics (True-semantics), e a semântica estrutural.

\section{O CASO DO FRAME SELF_MOTION}

Nesta seção, apresenta-se o estudo contrastivo das unidades lexicais evocadoras do frame Self motion em inglês e português, salientando as divergências entre as unidades lexicais em inglês e português.

\subsection{O frame Self_motion em Inglês e Português}

Segundo a FrameNet, o frame Self_motion descreve uma situação em que "o self mover, um ser vivo, se move ao longo de um percurso por meio das suas próprias forças". ${ }^{11}$ Dentre os 115 verbos que são apontados pela FrameNet como evocadores do frame Self_motion, encontram-se verbos como parade, run, swim e walk. Ao se buscar equivalentes para os 115 verbos em inglês apontados na FrameNet como evocadores do frame em questão, obtiveram-se equivalentes para apenas 29 verbos.

Se, por um lado, obteve-se sucesso em encontrar equivalentes para jump (pular), walk (caminhar), swim (nadar) e parade (desfilar), por outro lado, enfrentou-se a dificuldade na busca pelos equivalentes de verbos como wade, creep e bustle. Wade associa-se à ideia de caminhar com dificuldade na água; creep, à ideia de mover-se furtivamente e bustle, à de se mover energicamente. O português não dispõe de construções similares para expressar tais formas de movimento. Completam a lista dos verbos sem equivalentes em português os verbos amble, wander, hike, jog, limp e scurry.

Esta dificuldade em se encontrarem equivalentes em português para verbos de movimento em inglês suscita diferentes perguntas: (a) Que fatores estão implicados neste comportamento? (b) Por que português e inglês contrastam no que tange à exemplificação de verbos que compõem oframe Self_motion? (c)

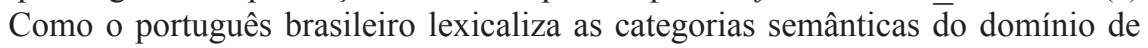
movimento?

\footnotetext{
${ }^{11}$.https://framenet2.icsi.berkeley.edu/fnReports/data/frameIndex.xml?frame=Self_motion
} 
De acordo com Talmy (1985), as línguas se dividem em dois grupos tipológicos: as línguas do tipo sattelite-framed e as línguas do tipo verb-framed. Esta distinção considera a forma como a propriedade central de um evento de movimento, isto é, a categoria percurso (path), se expressa linguisticamente e põe o foco nas noções de verbo principal e satélites. As línguas alocadas no primeiro grupo, como é o caso do inglês, parecem codificar a informação de percurso em satélites como out, into, down, como em run out, jump into e crawl down. As línguas do segundo grupo, exemplificados pelo espanhol e português, seguem a estratégia oposta: a informação de percurso não é codificada em um elemento separado, mas costuma se incorporar ao verbo. Descer (go down) e entrar (come in) ilustram esta tendência. A codificação do componente é considerada como opcional nas línguas do tipo verb-framed e costuma se expressar em um elemento separado. A sequência sair correndo (go out running) exemplifica este comportamento.

Para explorar a natureza de tal tipologia, Talmy (1985) identificou cinco componentes para descrever um evento de movimento: o movimento propriamente dito, a figura (o objeto em movimento), a base (a fonte, alvo ou locação do movimento), o percurso (o curso seguido pela figura), a maneira (a forma como a figura se move) e a causa (se o movimento é agentivo ou não). Em Inglês, os componentes de maneira e movimento são tipicamente incorporados por uma partícula (ou satélite): The girl ran out of the house. Em Espanhol, em contrapartida, o verbo principal tende a incorporar o movimento e o percurso do evento, enquanto a maneira de movimento se expressa como um gerúndio ou sintagma preposicional: La nina entró a la casa corriendo/ The girl entered the house running. A lexicalização do componente maneira no verbo principal de uma oração é uma característica de línguas como o inglês e tem motivado estudos sobre as propriedades semânticas dos verbos e seu efeito sobre o comportamento de padrões verbais.

\subsection{O verbo to walk e seus equivalentes}

A diferença em números de verbos evocadores do frame Self_motion parece indicar que o português brasileiro, assim como o espanhol, aloca-se no grupo das línguas do tipo verb-framed. Enquanto o inglês dispõe de uma vasta lista de verbos incorporando o componente maneira na raiz do verbo, o português - haja vista a dificuldade de encontrarmos equivalência - parece comportar-se como o espanhol, à medida que se vale de construções gerundivas para expressar o componente maneira.

A partir da consulta ao corpus COMPARA ${ }^{12}$, é possível se estabelecer os equivalentes mais recorrentes para o verbo to walk. As ocorrências contendo o verbo to walk e suas traduções em português possibilitam verificar como ocorre a lexicalização dos componentes semânticos estudados, em especial, os componentes percurso e maneira. Foram coletados 210 pares de sentenças.

12. Maiores informações em http://www.linguateca.pt 
Cadernos de ESTUDOS LINGǘsTIICOS (55.1) - Jan./Jun. 2013

\begin{tabular}{|c|c|}
\hline Equivalentes de walk & Número de Ocorrências \\
\hline Caminhar & 59 \\
\hline Andar & 64 \\
\hline Passear & 9 \\
\hline Outros & 78 \\
\hline
\end{tabular}

Quadro 1: Equivalentes de Tradução

A amostra extraída do COMPARA traz indicações reveladoras no que diz respeito à tipologia proposta por Talmy (1985). O quadro 1 mostra que caminhar $\mathrm{e}$ andar são os equivalentes de tradução mais recorrentes, confirmando os resultados provenientes das consultas aos recursos lexicográficos. Contudo, chama a atenção também o número de ocorrências indicadas como Outros, o que, em termos de equivalentes, vem a corresponder a diferentes equivalentes, como ilustrado nos pares seguintes.

(1) a. I daresay a lot of other people driving past felt the same way, but unlike them I knew I could walk into the showroom and buy the car without even having to think if I could afford it.

b. Devo dizer que muitos outros que passavam também sentiam a mesma pontada de desejo, mas sabia que só eu poderia entrar no showroom e comprar o carro sem pensar duas vezes.

(2) a. Uncle Maximino dying and he walked into the room.

b. Tio Maximino morrendo, ele chegava no quarto.

(3) a. When night closes perfectly around me, no moon or stars, no delights, nothing at all, I jump from the stone and walk down the dirt track into the farm.

b. Quando a noite se consuma, perfeita, sem lua nem estrelas, sem encantos, sem nada, salto da pedra e vou descendo a estradinha de terra batida sítio adentro.

Estes exemplos ilustram uma tendência observada no conjunto de 78 pares representando a categoria Outros: a semântica de movimento expressar-se por meio de partículas ou satélites. Nos exemplos acima, tem-se walk into e walk down. Pode-se ainda mencionar as partículas towards, in, back round, through, $u p$, by, to, acompanhando o verbo walk, assim como a diversidade de equivalentes em português.

\section{O CASO DO FRAME CRIMINAL_PROCESS}

Nesta seção, discutem-se as divergências entre os equivalentes em português das unidades lexicais arraignment, charge, indictment e try, unidades 
CHISHMAN e BERTOLDI - A semântica de frames como paradigma...

lexicais evocadores de diferentes subframes que integram o frame Criminal_ porcess. ${ }^{13}$ Essa discussão, juntamente com a discussão apresentada na seção anterior sobre as divergências entre as unidades lexicais que evocam o frame Self_motion e seus equivalentes em português, embasará a discussão do uso da Semântica de Frames como paradigma de análise nos estudos multilíngues.

O caso do frame Criminal process é diferente do frame Self motion. Enquanto os padrões de lexicalização e de polissemia dos verbos de movimento do português diferem dos verbos de movimento do inglês, causando dificuldades para os estudos multilíngues, tais como a tradução e o desenvolvimento de recursos lexicais, as divergências entre unidades lexicais do inglês e seus equivalentes em português, no que diz respeito às unidades lexicais evocadoras dos subframes que compõem oframe Criminal_process, estão relacionadas a diferenças entre sistemas jurídicos.

As diferenças entre os sistemas jurídicos americano e brasileiro fazem com que o conceito (jurídico) representado por uma determinada unidade lexical (que também pode ser chamada de termo jurídico) seja diferente entre as duas línguas e realidades culturais em contraste. Assim, mesmo que uma unidade lexical em inglês tenha um equivalente em português, como é o caso de to charge/denunciar, o significado de ambas as unidades lexicais nas sociedades americana e brasileira, bem como a estrutura conceitual evocada pelas unidades lexicais, é diferente.

A equivalência de unidades lexicais entre línguas pode se tornar ainda mais difícil em domínios socialmente orientados, como o Direito. A dificuldade de se encontrar equivalentes em determinados casos pode ser percebida mesmo em dicionários bilíngues. O Noronha Dicionário Jurídico (GOYOS Jr., 1992) apresenta como a unidade lexical julgar como único equivalente em português da unidade lexical to try em inglês. Segundo a FrameNet, a unidade lexical to try evoca o frame Try_defendant ${ }^{14}$, que descreve uma situação em que "um RÉU é julgado por um JÚRI ou JUIZ em um TRIBUNAL por determinadas ACUSAÇÕES." ${ }^{\prime 5}$ Ao se comparar a unidade lexical to try e o frame Try_ defendant com o seu equivalente em português, percebe-se que tanto to try quanto julgar evocam o mesmo cenário: há um réu sendo julgado por alguma acusação contra si e cabe ao juiz ou ao júri a decisão sobre a culpa do réu. Neste caso, a estrutura conceitual evocada pela unidade lexical em inglês é semelhante à estrutura conceitual evocada pela unidade lexical equivalente em português. Neste caso, tem-se uma equivalência não apenas entre unidades lexicais de línguas diferentes, mas também uma equivalência de frames evocados.

Um segundo caso mais complexo é o caso das unidades lexicais to charge e to indict, evocadoras do frame Notification_of_charges. O Noronha (2011).

13. Um estudo mais detalhado do frame Criminal_process é apresentado em Bertoldi

14. Seguindo a metodologia adotada pelos diferentes grupos de pesquisas que trabalham com a FrameNet, os nomes dos frames são grafados em Courier New e os nomes dos elementos de frame (os participantes dos eventos) são escritos em caixa alta.

15. "A Defendant is tried by a Jury or Judge in a Court for Charges" (https://framenet2.icsi. berkeley.edu/fnReports/data/frameIndex.xml?frame=Try_defendant). 
Dicionário Jurídico aponta acusar como equivalente em português de to charge e pronunciar como equivalente de to indict. A existência de um equivalente de dicionário para as unidades lexicais to charge e to indict sugere, a princípio, uma equivalência no significado dessas unidades lexicais com seus equivalentes em português. No entanto, ao se comparar o frame que é evocado pelas unidades lexicais to charge e to indict com os frames que são evocados pelos seus equivalentes de dicionário, percebe-se que os cenários se diferenciam.

Enquanto to charge e to indict evocam um cenário em que um suspeito é acusado formalmente de um crime, a unidade lexical acusar aponta para um cenário em que um suspeito é acusado formalmente de um crime por um promotor e a unidade lexical pronunciar aponta para um cenário em que um juiz decide se a acusação apresentada pelo promotor é procedente. Neste caso, o juiz pronuncia o acusado, que será levado a julgamento. Portanto, diferente das unidades lexicais to charge e to indict, que evocam o mesmo frame, a unidade lexical acusar evoca um frame denúncia; já a unidade lexical pronunciar evoca um frame de pronúncia. Denúncia e pronúncia representam etapas diferentes em um processo penal no Brasil.

A unidade lexical arraignment, por sua vez, representa um caso de falta total de equivalência. Segundo a FrameNet, o frame Arraignment descreve uma situação em uma audiência no tribunal em que "um réu é informado das acusações contra si, faz uma declaração e o juiz toma uma decisão, bem como a fiança correspondente." 16 O frame Arraignment é evocado pelas unidades lexicais arraign.v e arraignment.v. A audiência de arraignment é uma etapa do processo penal americano que não possui correspondência no processo penal brasileiro. Considerando que o cenário descrito pelo frame Arraignment não faz parte da realidade cultural brasileira, nem frame, nem unidades lexicais possuem correspondentes em português. Essa falta de correspondência pode ser verificada nos recursos lexicais. Ao procurar-se pela unidade lexical arraingment no Noronha Dicionário Jurídico, encontra-se apenas uma paráfrase que tenta explicar, através do conhecimento jurídico brasileiro, um conhecimento específico do sistema jurídico americano: "leitura do libelo na presença do acusado". O arraignment é muito mais do que a leitura da acusação na presença do acusado. Durante o arraignment o acusado declara sua culpa ou inocência. O dicionarista, ao tentar encontrar equivalência para uma palavra que descreve um evento jurídico que não possui correspondência no sistema jurídico da língua alvo, pode cometer erros que prejudicam a qualidade da informação contida nos recursos lexicais. Neste ponto a Semântica de Frames pode contribuir com os estudos multilíngues e para a criação de recursos lexicais multilíngues mais consistentes.

16. "At a court hearing, a Defendant is informed of the Charges against him or her, (usually) enters a plea, and a decision is made by a Judge as to the amount of bail, if any" (https://framenet2.icsi. berkeley.edu/fnReports/data/frameIndex.xml?frame=Arraignment). 


\section{A EQUIVALÊNCIA SOB O VIÉS DA SEMÂNTICA DE FRAMES}

A Semântica de Frames pode auxiliar no entendimento de fenômenos linguísticos relacionados à multilingualidade. Questões relacionadas aos diferentes padrões de polissemia entre línguas (ALTENBERG; GRANGER, 2002) e diferenças entre as realidades culturais (DUVAL, 2008[1991]) podem ser explicitados por meio da teoria de frames semânticos. A polissemia do verbo to walk na língua-fonte pode ser percebida por meio da diversidade de equivalentes na língua portuguesa: caminhar, andar, passear. A polissemia dos itens lexicais também não é correspondente entre o inglês e o português. Enquanto o verbo to walk traz consigo o sentido de "movimento realizado por um ser que se move por conta própria", podendo ser relacionado ao frame Self_motion na FrameNet, o seu equivalente andar em português é polissêmico, podendo ser relacionado aos frames Motion e Self_motion.

As diferenças tipológicas entre as línguas sattelite-framed e verb-framed (TALMY, 1985), expressas através dos padrões de lexicalização dos verbos de movimento em inglês e português também impõem desafios para os estudos multilíngues. Verbos como run out exemplificam o que Snell-Hornby (1983) chama de cobertura parcial. Parte do significado do verbo é expressa em português por meio de um verbo, enquanto o restante do significado necessita ser parafraseado por meio de uma expressão gerundiva: sair correndo.

No caso das unidades lexicais relacionadas ao domínio jurídico, as diferenças entre as realidades culturais (DUVAL, 2008[1991]) geram diferentes graus de equivalência. As unidades lexicais relacionadas ao domínio jurídico podem apresentar uma equivalência total, como no caso das unidades lexicais to try e julgar. Essas unidades lexicais também podem apresentar equivalência, porém diferindo no seu significado, dada a realidade cultural que essas unidades lexicais representam, como é o caso da unidade lexical to charge e seu equivalente acusar em português. Em outros casos, como da unidade lexical arraignment, a realidade cultural expressa pela unidade lexical é tão especializada, que não há equivalente na língua-alvo.

Os frames semânticos, por representarem estruturas esquematizadas de conhecimento, podem ser importantes nos estudos multilíngues. Essas estruturas esquematizadas de conhecimento ajudam a identificar o significado das unidades lexicais. A unidade lexical acusar, por exemplo, em muitos dicionários, é dada como equivalente de to charge. No entanto, ao se analisar o frame evocado pela unidade lexical em inglês e pela unidade lexical em português, pode-se ver que, apesar da haver uma equivalência de trabalho entre as unidades lexicais to charge e acusar, o conhecimento evocado em inglês pela unidade lexical to charge referese a um conhecimento socialmente compartilhado por uma sociedade, enquanto que o conhecimento evocado pela unidade lexical em português se refere a um conhecimento compartilhado por outra sociedade. A importância da Semântica de Frames é reconhecida inclusive para a tradução. Snell-Hornby (1988; 2005) postula que o entendimento dos frames semânticos na língua-fonte ajuda o tradutor a realizar uma tradução mais verossímil. 


\section{CONSIDERAÇÕES FINAIS}

Nosso interesse neste estudo consistiu em refletir sobre o potencial descritivo e explanatório da Semântica de Frames (e consequentemente o arcabouço da FrameNet) para tratar de questões de multilingualidade. É importante destacar que tal temática vem sendo explorada pela comunidade internacional, especialmente no que tange à construção de recursos computacionais multilíngues; contudo, os aspectos linguísticos envolvidos, como é o caso dos padrões de lexicalização, equivalência de tradução e polissemia, ainda carecem de aprofundamento e de metodologia.

Através dos estudos contrastivos aqui apresentados, pudemos nos certificar de que a Semântica de Frames, ao permitir que diferentes significados de uma palavra sejam associados a frames distintos, tem muito a contribuir especialmente no desenvolvimento de recursos lexicais. A orientação cognitiva da Semântica de Frames também ajuda a explicar os diferentes níveis de equivalência das unidades lexicais, especialmente quando as divergências de sentido entre as línguas estão associadas às realidades culturais próprias de cada sociedade evocadas pelas unidades lexicais.

\section{REFERÊNCIAS BIBLIOGRÁFICAS}

ALTERNBERG, B.; GRANGER, S. (2002). Recent trends in cross-linguistic lexical studies. In: ALTERNBERG, B.; GRANGER, S. (Eds.). Lexis in Contrast: Corpus-based Approaches. Amsterdam: John Benjamins, p 3-48.

BERTOLDI, A. (2011). Os Limites da Criação Automática de Léxicos Computacionais Baseados em Frames: Um Estudo Contrastivo do Frame Criminal_process. In: The 8th Brazilian Symposium in Information and Human Language Tecnology - STIL2011, 2011, Cuiabá. The 8th Brazilian Symposium in Information and Human Language Tecnology - STIL2011. Cuiabá: Universidade Federal do Mato Grosso, p. 87-96.

BOAS, H. C. (2002). Bilingual FrameNet Dictionaries for Machine Translation. In: Proceedings of the Third International Conference on Language Resources and Evaluation. Las Palmas, Spain. Vol. IV: 1364-1371.

Semantic Frames as Interlingual Representations for Multilingual Lexical Databases. International Journal of Lexicography. 2005 18(4): 445-478.

(2009). (Ed.) Multilingual FrameNets in computational lexicography - methods and applications. Berlin/New York: Mouton de Gruyter, 352p.

CRUSE, D. A. (1986). Lexical semantics. New York: Cambridge University Press, 310p.

DICIONÁRIO INGLÊS-PORTUGUÊS. (2000). Antônio Houais, editor, Ismael Cardim, co-editor. $11^{\mathrm{a}}$ ed. Rio de Janeiro: Record, 928p.

DUVAL, A. (2008). Equivalence in bilingual dictionaries. In: FONTENELLE, T. (Ed.) Practical lexicography: a reader. Oxford: Oxford University Press [1991], p.273-282.

FILLMORE, C. J. (1968). The case for case. In: BACH, E.; HARMS, R. T. (Eds.) Universals in Linguistic Theory. Volume 67. New York: Holt, Rinehart and Winston, p.1-88. 
CHISHMAN e BERTOLDI - A semântica de frames como paradigma...

(1977). Scenes-and-frames semantics. In ZAMPOLLI, A. (Ed.): Linguistic Structures Processing: Fundamental Studies in Computer Science, No. 59, North Holland Publishing, p.55-88.

(1982). Frame semantics. In: The Linguistic Society of Korea (Eds.). Linguistics in the Morning Calm. Seoul: Hanshin, p.111-37.

. (1985). Frames and the semantics of understanding. Quaderni di Semantica. Vol.6, N.2, p.222254.

. ; JOHNSON, C. R.; PETRUCK, M. R. L. (2003). Background to FrameNet. International Journal of Lexicography. Vol.16, $\mathrm{N}^{\mathrm{o}} .3, \mathrm{p} .235-250$.

LONGMAN DICTIONARY OF CONTEMPORARY ENGLISH. Third edition. (1995). Essex: Longman.1668p.

GOLDBERG, A. (1995). Constructions. a construction grammar approach to argument structure. Chicago: University of Chicago Press. 271p.

- (2002). Surface generalizations: an alternative to alternations. In: Cognitive Linguistics, Vol.13, No.4, p.327-356.

GOYOS Jr., D. N. (1992). Noronha's Legal Dictionary - Noronha Dicionário Jurídico: EnglishPortuguese, Portuguese-English - Inglês-Português, Português-Inglês. 1.ed. São Paulo: Observador Legal.

PETRUCK, M. (1996). Frames semantics. In: ÖSTMAN, J-O; VERSCHUEREN, J.; BLOMMAERT, J. (Eds.) Handbook of pragmatics. Amsterdam/Philadelphia: John Benjamins.

RAPPAPORT, M.; LEVIN, B. (2001). An event structure account of English resultatives. In: Language 77, p.766-797.

OHARA, K. H. (2009). Frame-based contrastive lexical semantics in Japanese FrameNet: The case of risk and kakeru. In: BOAS, H. C. (Ed.) Multilingual FrameNets in computational lexicography: Methods and applications. Berlin/New York: Mouton de Gruyter, p.163-182.

SALOMÃO, M. M.M. (2009). FrameNet Brasil: um trabalho em progresso. Calidoscópio. Vol.7, No.3, p.171-182.

SNELL-HORNBY, M. (1983). Verb-descriptivity in German and English: A contrastive study in semantic fields. Heidelberg: Carl Winter - Universitätsverlag, 279p.

(1988). Tranlation studies: an integrated approach. Amsterdam/Philadelphia: Benjamins Publishing Company, 163p.

. (2005). "Of catfish and blue bananas: scenes-and-frames semantics as a contrastive 'knowledge system' for translation.' In: DAM, V.; ENGBERG, J.; GERZYMISCH-ARGBOGAST, H. (eds.) Knowledge systems and translation. Berlin/New York: Mouton de Gruyter, p. 193-206.

SUBIRATS, C. (2009). Spanish FrameNet: A frame-semantic analysis of the Spanish lexicon. In: BOAS, H. C. (Ed.) Multilingual FrameNets in computational lexicography: Methods and applications. Berlin/New York: Mouton de Gruyter, p.136-162.

TALMY, L. (1985). Lexicalization patterns. In: SHOPEN, T. (Ed.) Language typology and syntactic description. Cambridge: Cambridge University Press, p. 57-147.

VANNEREM, M.; SNELL-HORNBY, M. (1986). Die Szene hinter dem Text: 'scenes-and-frames semantics' in der Übertzung. In: SNELL-HORNBY (ed.). Übersetzungswissenschaft - Eine Neuorientierung. Zur Integrierung von Theorie und Praxis (UTB 1415). Tübingen: Francke, p.184-205. 
Cadernos de EsTUDOS LINGǘsTTCOS (55.1) - Jan.JJun. 2013

VERMEER, H.; WITTE, H. (1990). Mögen Sie Zistrosen? Scenes \& frames \& channels im translatorishen Handeln. Heidelberg: Groos, 165p. 
